[][]$\longrightarrow$
[][] $\mathrm{S}$
[][] $\mathrm{J}$
[][] $\mathrm{W}$
[][] $\mathrm{B}$
[]$[\mathrm{K}(\mathrm{H})$
[]$[\mathrm{C}$
[][]$^{+}$
[][] $\mathrm{P}(\mathrm{E})$
[][] $\mathrm{L}$
$[1][]|1|$




\title{
Stability with respect to a part of the variables of stochastic dierential equations driven by G-Brownian motion
}

\author{
Tomas Caraballo ${ }^{1}$, Faten Ezzine ${ }^{2}$, and Mohamed Ali Hammami ${ }^{2}$ \\ ${ }^{1}$ Universidad de Sevilla \\ ${ }^{2}$ University of Sfax Faculty of Sciences of Sfax
}

May 6, 2020

\begin{abstract}
In this paper, we investigate the pth moment exponential stability of stochastic differential equations driven by G-Brownian motion (G-SDEs) with respect to a part of the variables by means of the G-Lyapunov functions and Gronwall's inequalities. We establish sufficient conditions to ensure the quasi sure exponential stability of stochastic differential equations perturbed by G-Brownian motion with respect to a part of the variables. Some illustrative examples to show the usefulness of the stability with respect to a part of variables notion are also provided.
\end{abstract}

Keywords: G-Stochastic differential equations, G-Itô formula, G-Brownian motion, pth moment exponential stability with respect to a part of the variables, quasi sure exponential stability with respect to a part of the variables .

Mathematics Subject Classification Primary 93E03 · Secondary 60H10.

\section{Introduction}

Stability of stochastic differential equations (SDEs) has become a very prevalent theme of recent research in Mathematics and its applications. Stochastic systems are used to model problems from the real world in which some kind or randomness or noise must be taken into account.

Some stochastic models cannot be proved to fulfill stability properties with respect all the unknown variables of the system. However, it is very interesting in some situations to analyze if it is still possible to prove some stability properties with respect to some of the variables in the problem. It is worth mentioning that, recently considerable attention has been paid to the concept of stability with respect to a part of the system states. Such concept arises from the study of combustion systems (E. Awad, n.d.), vibrations in rotating machinery (K. Y. Lum, n.d.), biocenology (N. Rouche, n.d.), inertial navigation systems (Sinitsyn, n.d.), electro-magnetics (V. I. Zubov, 1982), and spacecraft stabilization via gimballed gyroscopes and/or flywheels (V. I. Vorotnikov, n.d.).

The method of Lyapunov functions is one of the most powerful tool to study the stability of stochastic dynamical systems. Lyapunov stability of stochastic dynamical systems has attracted the attention of several authors, we would like to mention here the references (missing citation; missing citation; R. Z. Has'minskii, 1980; Mao, n.d.), among others.

With the emergence of the second method of Lyapunov as an essential means in science, engineering, and applied mathematics, numerous exciting and important variants to Lyapunov's original concept stability were proposed. 
One of these involves the notion of stability with respect to a part of the variables, Peiffer and Rouche (1969), Rouche et al. (N. Rouche, n.d.), Rumyantsev (1957), Rumyantsev and Oziraner (V, n.d.), Savchenko and Ignatyev (1989), Vorotnikov (V. I. Vorotnikov, n.d.), Vorotnikov and Rumyantsev (V. I. Vorotnikov \& Control with Respect to a Part of the Phase Coordinates of Dynamic Systems: Theory, n.d.). This type of stability has been used in investigating the qualitative properties of equilibria and boundedness properties of motions of dynamical systems determined by ordinary differential equations, difference equations, functional differential equations, stochastic differential equations, etc. It involves a notion of stability with respect to only a prespecified subset of the state variables characterizing the motions of the system under investigation.

For the dormant applications in uncertainty problems, risk measures, and superhedging in finance, considerable attention has been paid to the theory of nonlinear expectation. Notably, Peng (2006) built the fundamental theory of time-consistent G-expectation and G-conditional expectation, where G is the infinitesimal generator of a nonlinear heat equation. Under the G-framework, Peng (1002; 2006) introduced the notion of G-normal disribution, G-Brownian motion and he also established the corresponding stochastic calculus of Itô's type. Since then, many researches have been carried out on the stochastic analysis with respect to the G-Brownian motion. On that basis, Gao (F. Gao \& homomorphic flows for stochastic differential equations driven by G-Brownian, n.d.) and Peng (1002) studied the existence and uniqueness of the solution of G-SDE under a standard Lipschitz condition. Moreover, Lin (Y. Lin, n.d.) obtained the existence and uniqueness of the solution of G-SDE with reflecting boundary. The G-Brownian motion has a very rich and interesting new structure which non-trivially, for a recent account and development of this theory we refer the reader to see (X. Bai \& uniqueness of solutions to stochastic differential equations driven by G-Brownian, n.d.),(missing citation), and (2013).

However, so far no work have been reported about stability with respect to a part of variables of G-stochastic differential equations. Consequently, this paper is devoted to establishing some criteria for the $p$-th moment exponential stability and the quasi sure exponential stability with respect to a part of the variables of GSDEs by means of the G-Lyapunov functions method and Gronwall inequalities.

The content of this paper is organized as follows: In Section 2, we recall some necessary preliminaries and results. In Section 3, we establish sufficient conditions to ensure $p$-th moment exponential stability and quasi sure exponential stability with respect to a part of the variables of stochastic differential equations driven by G-Brownian motion (GSDEs, in short) by using the G-Lyapunov techniques. In section 4, we give sufficient conditions of quasi sure exponential stability with respect to a part of the variables of G-stochastic perturbed systems based on Gronwall's inequalities. Moreover, we exhibit some illustrative examples to show the applicability of our abstract theory.

\section{Preliminaries}

In this section, we briefly recall some notations and preliminaries about sublinear expectations and G-Brownian motion. For more details, one can refer to (P. Luo \& ordinary differential equations, 2014; 1002; 2006; S. Peng \& related stochastic calculus under G-expectation, 2008).

\section{Notations on G-stochastic calculus}

- $\mathbb{R}^{n}$ : the space of $n$-dimensional real column vectors.

- $\langle x, y\rangle$ : the scalar product of two vectors $x, y \in \mathbb{R}^{n}$.

- |.| : arbitrary spacial norm.

- $\mathcal{B}(\Omega)$ : the Borel $\sigma$-algebra of $\Omega$.

- $\mathcal{C}_{b, \operatorname{Lip}\left(\mathbb{R}^{n}\right)}$ : the space of all bounded real-valued Lipshitz continuous functions.

- $\mathbb{L}^{0}$ : the space of all $\mathcal{B}(\Omega)$-measurable real functions. 
- $M_{G}^{p, 0}=\left\{\zeta_{t}:=\sum_{j=0}^{N-1} \zeta_{j} 1_{\left[t_{j}, t_{j+1}[\right.}: \zeta_{j} \in \mathbb{L}_{G}^{p}\left(\omega_{j}\right)\right\}$.

- $M_{G}^{p}(0, T)$ : the completion of $M_{G}^{p, 0}$ under $\|\cdot\|_{M_{G}^{p}}$.

Let $\Omega$ be a given set and let $\mathcal{H}$ be a linear space of real valued defined on $\Omega$. We further suppose that $\mathcal{H}$ satisfies $a \in \mathcal{H}$ for each constant $a$ and $|X| \in \mathcal{H}$ if $X \in \mathcal{H}$.

Definition 0.1. A sublinear expectation $\widehat{\mathbb{E}}$ on $\mathcal{H}$ is a functional $\widehat{\mathbb{E}}: \mathcal{H} \rightarrow \mathbb{R}$ satisfying the following properties: for all $X, Y \in \mathcal{H}$,

i) Monotonicity: if $X \geq Y$, then $\widehat{\mathbb{E}}[X] \geq \widehat{\mathbb{E}}[Y]$.

ii) Constant preserving: $\widehat{\mathbb{E}}[a]=a, \forall a \in \mathbb{R}$.

iii) Sub-additivity: $\widehat{\mathbb{E}}[X+Y] \leq \widehat{\mathbb{E}}[X]+\widehat{\mathbb{E}}[Y]$.

iv) Positive homogeneity: $\widehat{\mathbb{E}}[\lambda X]=\lambda \widehat{\mathbb{E}}[X], \quad \lambda \geq 0$.

The triple $(\Omega, \mathcal{H}, \widehat{\mathbb{E}})$ is called a sublinear expectation space. $X \in \mathcal{H}$ is called a random variable in $(\Omega, \mathcal{H}, \widehat{\mathbb{E}})$. $Y=\left(Y_{1}, \ldots, Y_{n}\right)$, where $Y_{i} \in \mathcal{H}$ is called a n-dimensional random vector in $(\Omega, \mathcal{H}, \widehat{\mathbb{E}})$.

Definition 0.2. Let $X_{1}$ and $X_{2}$ be two n-dimensional random vectors defined on sublinear expectation spaces $\left(\Omega_{1}, \mathcal{H}_{1}, \widehat{\mathbb{E}}_{1}\right)$ and $\left(\Omega_{2}, \mathcal{H}_{2}, \widehat{\mathbb{E}}_{2}\right)$, respectively. They are called identically distributed, denoted by $X_{1} \stackrel{d}{=} X_{2}$, if

$$
\widehat{\mathbb{E}}_{1}\left[\varphi\left(X_{1}\right)\right]=\widehat{\mathbb{E}}_{2}\left[\varphi\left(X_{2}\right)\right], \quad \forall \varphi \in \mathcal{C}_{b, \operatorname{Lip}\left(\mathbb{R}^{n}\right)} .
$$

$\bar{X}$ is said to be an independent copy of $X$ if $\bar{X} \stackrel{d}{=} X$ and $\bar{X}$ is independent from $X$.

Definition 0.3. (G-Normal Distribution) A random variable $X$ on a sublinear expectation space $(\Omega, \mathcal{H}, \widehat{\mathbb{E}})$ is called G-normal distributed, if for any $a, b \geq 0$

$$
a X+b \bar{X} \stackrel{d}{=} \sqrt{a^{2}+b^{2}} X
$$

where, $\bar{X}$ is an independent copy of $X$.

Let $\Omega$ be the space of all $\mathbb{R}^{d}$-valued continuous paths $\left(\omega_{t}\right)_{t \geq 0}$ with $\omega(0)=0$. We assume moreover that $\Omega$ is a metric space equipped with the following distance:

$$
\rho\left(\omega^{1}, \omega^{2}\right):=\sum_{N=1}^{\infty} 2^{-N}\left(\max _{0 \leq t \leq N}\left(\left|\omega_{t}^{1}-\omega_{t}^{2}\right|\right) \wedge 1\right),
$$

and consider the canonical process $B_{t}(\omega)=\omega(t), t \in[0, \infty)$, for $\omega \in \Omega$; then for each fixed $T \in[0, \infty)$, we have

$$
L_{i p}^{0}\left(\Omega_{T}\right):=\left\{\varphi\left(B_{t_{1}}, B_{t_{2}}, \ldots, B_{t_{n}}\right): n \geq 1,0 \leq t_{1} \leq \ldots \leq t_{n} \leq T, \varphi \in C_{b, l i p}\left(\mathbb{R}^{d \times n}\right)\right\} .
$$

Definition 0.4. On the sublinear expectation space $\left(\Omega, L_{i p}^{0}\left(\Omega_{T}\right), \widehat{\mathbb{E}}\right)$, the canonical process $\left(B_{t}\right)_{t \geq 0}$ is called a G-Brownian motion, if the following properties are satisfied:

(i) $B_{0}=0$.

(ii) for $t, s \geq 0$, the increment $B_{t+s}-B_{t} \stackrel{d}{=} \sqrt{s} X$, where $X$ is G-normal distributed.

(iii) for $t, s \geq 0$, the increment $B_{t+s}-B_{t}$ is independent from $\left(B_{t_{1}}, B_{t_{2}}, \ldots, B_{t_{n}}\right)$ for each $n \in \mathbb{N}$, and $0 \leq t_{1} \leq t_{2} \leq \ldots \leq t_{n} \leq t$.

Moreover, the sublinear expectation $\widehat{\mathbb{E}}[$.$] is called G-expectation.$

Let $\left(B_{t}\right)_{t \geq 0}$ be a 1-dimensional G-Brownian motion. The letter $G$ denotes the function

$$
G(a):=\frac{1}{2} \widehat{\mathbb{E}}\left[a B_{1}^{2}\right]=\frac{1}{2}\left(\bar{\sigma}^{2} a^{+}-\underline{\sigma}^{2} a^{-}\right), \quad a \in \mathbb{R},
$$

with, $\underline{\sigma}^{2}:=-\widehat{\mathbb{E}}\left[-B_{1}^{2}\right] \leq \widehat{\mathbb{E}}\left[B_{1}^{2}\right]:=\bar{\sigma}^{2}, 0 \leq \underline{\sigma} \leq \bar{\sigma}<\infty$. 
(Recall that $a^{+}=\max \{0, a\}$ and $\left.a^{-}=-\min \{0, a\}\right)$.

Now, we introduce the natural choquet capacity.

Definition 0.5. Let $\mathcal{B}(\Omega)$ the borel $\sigma$-algebra and $\mathcal{P}$ be a weakly compact collection of probability measures $P$ defined on $(\Omega, \mathcal{B}(\Omega))$, then the capacity $\hat{C}($.$) associated to \mathcal{P}$ is defined by

$$
\hat{C}(A):=\sup _{P \in \mathcal{P}} P(A), \quad A \in \mathcal{B}(\Omega) .
$$

Definition 0.6. A set $A \subset \mathcal{B}(\Omega)$ is polar if $\hat{C}(A)=0$. A property holds "quasi-surely" (q.s.) if it holds outside a polar set.

Lemma 0.1. (2010) Let $\left\{A_{k}\right\} \subset \mathcal{B}(\Omega)$ such that

$$
\sum_{k=1}^{\infty} \hat{C}\left(A_{k}\right)<\infty
$$

Then, $\lim _{k \rightarrow \infty} \sup A_{k}$ is polar.

Lemma 0.2. (2010) Let $X \in \mathbb{L}^{0}(\Omega)$ satisfying $\widehat{\mathbb{E}}|X|^{p}<\infty$, for $p>0$. Then, for each $M>0$,

$$
\hat{C}(|X|>M) \leq \frac{\widehat{\mathbb{E}}|X|^{p}}{M^{p}} .
$$

Lemma 0.3. (F. Gao \& homomorphic flows for stochastic differential equations driven by G-Brownian, n.d.) For each $p \geq 1, \eta \in M_{G}^{p}(0, T)$, and $0 \leq s \leq t \leq T$. Then,

$$
\widehat{\mathbb{E}}\left[\sup _{s \leq u \leq t}\left|\int_{s}^{u} \eta_{r} d\left\langle B^{a}, B^{\bar{a}}\right\rangle_{r}\right|^{p}\right] \leq\left(\frac{\sigma_{(a+\bar{a})+(a+\bar{a})^{T}}+\sigma_{(a-\bar{a})+(a-\bar{a})^{T}}}{4}\right)^{p}|t-s|^{p-1} \int_{s}^{t} \widehat{\mathbb{E}}\left[\left|\eta_{u}\right|^{p}\right] d u .
$$

Lemma 0.4. (F. Gao $\&$ homomorphic flows for stochastic differential equations driven by G-Brownian, n.d.) Let $p \geq 2, \eta \in M_{G}^{p}(0, T)$ and $0 \leq s \leq t \leq T$. Then, there exists some constant $C_{p}$ depending only on $p$ such that

$$
\widehat{\mathbb{E}}\left[\sup _{s \leq u \leq t}\left|\int_{s}^{u} \eta_{r} d B_{r}\right|^{p}\right] \leq C_{p}|t-s|^{\frac{p}{2}-1} \int_{s}^{t} \widehat{\mathbb{E}}\left[\left|\eta_{u}\right|^{p}\right] d u .
$$

\section{$p$-th moment exponential stability of G-stochastic differential equati- ons with respect to a part of the variables}

In this section, we aim to prove the pth moment exponential stability of stochastic differential equations driven by G-Brownian motion with respect to a part of the variables, via the G-Lyapunov functions.

Consider the following SDE driven by an m-dimensional G-Brownian motion:

$$
d x(t)=f(t, x(t)) d t+h(t, x(t)) d\langle B\rangle_{t}+g(t, x(t)) d B_{t}, \quad \forall x \in \mathbb{R}^{n}, \quad t \geq 0,
$$


where, $B_{t}=\left(B_{1}(t), \ldots ., B_{m}(t)\right)^{T}$ is an $m$-dimensional G-Brownian motion, and $(\langle B\rangle)_{t>0}$ is the quadratic variation process of $\mathrm{B}$.

We make a partition of the state $x:=\left(x_{1}, x_{2}\right) \in \mathbb{R}^{b} \times \mathbb{R}^{n-b}$, with $1 \leq b \leq n$. We obtain the following system:

$d x_{1}(t)=f_{1}\left(t, x_{1}(t), x_{2}(t)\right) d t+h_{1}\left(t, x_{1}(t), x_{2}(t)\right) d\langle B\rangle_{t}+g_{1}\left(t, x_{1}(t), x_{2}(t)\right) d B_{t}$

$d x_{2}(t)=f_{2}\left(t, x_{1}(t), x_{2}(t)\right) d t+h_{2}\left(t, x_{1}(t), x_{2}(t)\right) d\langle B\rangle_{t}+g_{2}\left(t, x_{1}(t), x_{2}(t)\right) d B_{t}$.

$(0.2)$

$$
f:=\left(f_{1}, f_{2}\right), h:=\left(h_{1}, h_{2}\right), g:=\left(g_{1}, g_{2}\right) .
$$

Assume that,

- $f_{1}, h_{1}$, and $g_{1} \in M_{G}^{2}\left([0, T], \mathbb{R}^{b}\right)$ satisfy the following condition: $\left|\phi_{1}\left(t, x_{1}, x_{2}\right)-\phi_{1}\left(t, y_{1}, x_{2}\right)\right| \leq K_{1}\left|x_{1}-y_{1}\right|, \quad$ for all $t \in[0, T], x_{1}, y_{1} \in \mathbb{R}^{b}, x_{2} \in \mathbb{R}^{n-b}$, $\phi_{1}=f_{1}, h_{1}$, and $g_{1}$ respectively, and $K_{1}$ is a positive constant.

- $f_{2}, h_{2}$, and $g_{2} \in M_{G}^{2}\left([0, T], \mathbb{R}^{n-b}\right)$ satisfy the following condition: $\left|\phi_{2}\left(t, x_{1}, x_{2}\right)-\phi_{2}\left(t, x_{1}, y_{2}\right)\right| \leq K_{2}\left|x_{2}-y_{2}\right|, \quad$ for all $t \in[0, T], x_{1} \in \mathbb{R}^{b}, x_{2}, y_{2} \in \mathbb{R}^{n-b}$, $\phi_{2}=f_{2}, h_{2}$, and $g_{2}$ respectively, and $K_{2}$ is a positive constant.

Under the precedent assumptions, there exists a unique global solution $x\left(t, t_{0}, x_{0}\right)=\left(x_{1}\left(t, t_{0}, x_{0}\right), x_{2}\left(t, t_{0}, x_{0}\right)\right)$ corresponding to the initial condition $x\left(t_{0}\right)=x_{0}=\left(x_{1_{0}}, x_{2_{0}}\right) \in \mathbb{R}^{n}$ (see S. Peng (1002; missing citation), Y. Ren, Q. Bi and R. sakthivel (2013), for more details).

In what follows we use $x\left(t, t_{0}, x_{0}\right)=\left(x_{1}\left(t, t_{0}, x_{0}\right), x_{2}\left(t, t_{0}, x_{0}\right)\right)$, or simply $x(t)=\left(x_{1}(t), x_{2}(t)\right)$ to denote a solution of our system on some small interval.

We assume that the origin $x=(0,0)$ is an equilibrium point of system $(0.2)$, that is $f_{i}(t, 0,0)=h_{i}(t, 0,0)=$ $g_{i}(t, 0,0)=0, \quad \forall t \geq t_{0} \geq 0,(i=1,2)$.

Definition 0.7. The equilibrium point $x=(0,0)$ of The G-SDE $(0.2)$ is said to be

(i) Pth moment exponentially stable with respect to $x_{1}$, if there exist positive constant $\lambda_{1}, \lambda_{2}$, and $p>0$ such that for all $x_{0} \in \mathbb{R}^{n}$, the following inequalities are satisfied:

$$
\widehat{\mathbb{E}}\left(\left|x_{1}\left(t ; t_{0}, x_{0}\right)\right|^{p}\right) \leq \lambda_{1}\left|x_{0}\right|^{p} e^{-\lambda_{2}\left(t-t_{0}\right)}, \quad \forall t \geq t_{0} \geq 0 .
$$


(ii) Quasi surely exponentially stable with respect to $x_{1}$, if

$$
\lim _{t \rightarrow \infty} \sup \frac{1}{t} \ln \left(\left|x_{1}\left(t, t_{0}, x_{0}\right)\right|\right)<0 \text {, q.s. }
$$

for all $x_{0} \in \mathbb{R}^{n}$.

Definition 0.8. The solution of the sub-system with respect to the variable $x_{2}$ is said to be quasi surely globally uniformly bounded, if for each $\alpha>0$, there exists $c=c(\alpha)>0$ (independent of $t_{0}$ ) such that,

$$
\text { for every } t_{0} \geq 0 \text {, and all } x_{2_{0}} \in \mathbb{R}^{n-b} \text { with } \quad\left|x_{2_{0}}\right| \leq \alpha, \quad \sup _{t \geq t_{0}}\left|x_{2}\left(t, t_{0}, x_{0}\right)\right| \leq c(\alpha), \quad \text { q.s. }
$$

where, $x_{2_{0}}=x_{2}\left(t_{0} ; t_{0}, x_{0}\right)$.

Definition 0.9. $C^{1,2}\left(\mathbb{R}_{+} \times \mathbb{R}^{n}, \mathbb{R}\right)$ is the family of all real-valued functions $V(t, x)$ defined on $\mathbb{R}_{+} \times \mathbb{R}^{n}$ which are twice continuously differentiable in $x$ and once in $t$.

If $V \in C^{1,2}\left(\mathbb{R}_{+} \times \mathbb{R}^{n}, \mathbb{R}\right)$, we define an operator L (called as G-Lyapunov function) from $\mathbb{R}_{+} \times \mathbb{R}^{n} \longrightarrow \mathbb{R}$ as follows:

$$
L V(t, x):=V_{t}(t, x)+V_{x} f(t, x)+G\left(\left\langle V_{x}(t, x), 2 h(t, x)\right\rangle+\left\langle V_{x x}(t, x) g(t, x), g(t, x)\right\rangle\right),
$$

where,

$V_{t}(t, x)=\frac{\partial V}{\partial t}(t, x) ; V_{x}(t, x)=\left(\frac{\partial V}{\partial x_{1}}(t, x), \frac{\partial V}{\partial x_{2}}(t, x)\right) ; V_{x x}(t, x)=\left(\frac{\partial^{2} V}{\partial x_{i} \partial x_{j}}(t, x)\right)_{n \times n}$.

By G-Itô's formula (S. Peng \& related stochastic calculus under G-expectation, 2008), it follows

$$
d V(t, x(t))=L V(t, x(t)) d t+V_{x}(t, x(t)) g(t, x(t)) d B_{t} .
$$

Our first main result in this section reads as follow.

Theorem 0.5. Assume that there exist $V \in C^{1,2}\left(\mathbb{R}_{+} \times \mathbb{R}^{n}, \mathbb{R}_{+}\right)$and positive constants $c_{i}(i=1,2,3)$, $p$, such that for all $t \geq t_{0} \geq 0$, and all $x=\left(x_{1}, x_{2}\right) \in \mathbb{R}^{n}$,

$$
c_{1}\left|x_{1}\right|^{p} \leq V(t, x) \leq c_{2}\left|x_{1}\right|^{p},
$$

$$
L V(t, x) \leq-c_{3}\left|x_{1}\right|^{p} .
$$


Furthermore, we suppose that $x_{2}\left(t, t_{0}, x_{0}\right)$ is quasi surely globally uniformly bounded.

Then, the trivial solution of the G-stochastic system (0.2) is pth moment exponentially stable with respect to $x_{1}$.

In order to prove this theorem we need to recall an important Lemma (2016):

Lemma 0.6. Let $\eta \in M_{G}^{1}(0, T)$ and $M_{t}=\int_{0}^{t} \eta(s) d\langle B\rangle_{s}-\int_{0}^{t} 2 G(\eta(s)) d s$.

Then, for each $t \in[0, T], \quad \widehat{\mathbb{E}}\left(M_{t}\right) \leq 0$.

Proof of Theorem 0.5. Applying G-Itô's formula to $e^{\frac{c_{3}}{c_{2}} t} V(t, x(t))$, we obtain

That is,

Where,

Taking, G-expectation on both sides, we get

On the other hand, since $\widehat{\mathbb{E}} \int_{t_{0}}^{t} e^{\frac{c_{3}}{c_{2}} s}\left\langle V_{x}(s, x(s)), g(s, x(s))\right\rangle d B_{s}=0$, and by Lemma $(0.6)$, we have $\widehat{\mathbb{E}}\left(M_{t}^{t_{0}}\right) \leq 0$. Consequently,

$$
\widehat{\mathbb{E}}\left(e^{\frac{c_{3}}{c_{2}} t} V(t, x(t))\right) \leq e^{\frac{c_{3}}{c_{2}} t_{0}} V\left(t_{0}, x\left(t_{0}\right)\right)+\widehat{\mathbb{E}} \int_{t_{0}}^{t} e^{\frac{c_{3}}{c_{2}}} s\left(\frac{c_{3}}{c_{2}} V(s, x(s))+L V(s, x(s))\right) d s
$$

This together with (0.5) and (0.6), implies 
That is,

$$
\widehat{\mathbb{E}}(V(t, x(t))) \leq c_{2} e^{-\frac{c_{3}}{c_{2}}\left(t-t_{0}\right)}\left|x_{1_{0}}\right|^{p}
$$

Due to (0.5) again and the fact that $\left|x_{1_{0}}\right| \leq\left|x_{0}\right|$, we deduce that

$$
\widehat{\mathbb{E}}\left|x_{1}\left(t, t_{0}, x_{0}\right)\right|^{p} \leq \frac{\widehat{\mathbb{E}}(V(t, x(t)))}{c_{1}} \leq \frac{c_{2}}{c_{1}} e^{-\frac{c_{3}}{c_{2}}\left(t-t_{0}\right)}\left|x_{0}\right|^{p}
$$

Therefore, the trivial solution of the G-stochastic system (0.2) is pth moment exponentially stable with respect to $x_{1}$.

Theorem 0.7. Assume that there exist $V \in C^{1,2}\left(\mathbb{R}_{+} \times \mathbb{R}^{n}, \mathbb{R}_{+}\right)$and positive constants $c_{i}(i=1,2,3), p$, such that for all $t \geq t_{0} \geq 0$, and all $x=\left(x_{1}, x_{2}\right) \in \mathbb{R}^{n}$,

$$
c_{1}\left|x_{1}\right|^{p} \leq V(t, x) \leq c_{2}\left|x_{1}\right|^{p}
$$

$$
L V(t, x) \leq\left(-c_{3}+\varphi(t)\right)\left|x_{1}\right|^{p}
$$

where $\varphi(t)$ is a continuous nonnegative function with

$$
\int_{0}^{+\infty} \varphi(t) d t \leq M<+\infty
$$

Furthermore, we suppose that $x_{2}\left(t, t_{0}, x_{0}\right)$ is quasi surely globally uniformly bounded.

Then, the trivial solution of the G-stochastic system (0.2) is pth moment exponentially stable with respect to $x_{1}$. 
In order to prove this theorem, we need to recall the following Gronwall Lemma (see, for instance, Dragomir (S. S. Dragomir \& applications, 2003)).

Theorem 0.8. Let $u(t)$ and $b(t)$ be nonnegative continuous functions for $t \geq \alpha$, and let

$$
u(t) \leq a+\int_{\alpha}^{t} b(s) u(s) d s, \quad t \geq \alpha,
$$

where $a \geq 0$ is a constant. Then,

$$
u(t) \leq a \exp \left(\int_{\alpha}^{t} b(s) d s\right), \quad t \geq \alpha
$$

Proof of Theorem (0.7). By using a similar reasoning as above we obtain,

$$
\widehat{\mathbb{E}}\left(e^{\frac{c_{3}}{c_{2}} t} V(t, x(t))\right) \leq e^{\frac{c_{3}}{c_{2}} t_{0}} V\left(t_{0}, x\left(t_{0}\right)\right)+\widehat{\mathbb{E}} \int_{t_{0}}^{t} e^{\frac{c_{3}}{c_{2}}} s\left(\frac{c_{3}}{c_{2}} V(s, x(s))+L V(s, x(s))\right) d s .
$$

On account of (0.7) and (0.8), we obtain

Hence,

$$
\widehat{\mathbb{E}}\left(e^{\frac{c_{3}}{c_{2}} t} V(t, x(t))\right) \leq e^{\frac{c_{3}}{c_{2}} t_{0}} V\left(t_{0}, x\left(t_{0}\right)\right)+\widehat{\mathbb{E}} \int_{t_{0}}^{t} \frac{\varphi(s)}{c_{1}} e^{\frac{c_{3}}{c_{2}} s} V(s, x(s)) d s .
$$

By Gronwall's inequality and condition (0.7), we obtain 
That is,

$$
\widehat{\mathbb{E}}(V(t, x(t))) \leq c_{2} e^{\frac{M}{c_{1}}}\left|x_{1_{0}}\right|^{p} e^{-\frac{c_{3}}{c_{2}}\left(t-t_{0}\right)}
$$

Due to the fact that $\left|x_{1_{0}}\right| \leq\left|x_{0}\right|$ and condition (0.7), we deduce from the last inequality that

$$
\widehat{\mathbb{E}}\left(\left|x_{1}(t)\right|^{p}\right) \leq \frac{c_{2}}{c_{1}} e^{\frac{M}{c_{1}}}\left|x_{0}\right|^{p} e^{-\frac{c_{3}}{c_{2}}\left(t-t_{0}\right)}
$$

Setting $\lambda_{1}=\frac{c_{2}}{c_{1}} e^{\frac{M}{c_{1}}}$ and $\lambda_{2}=\frac{c_{3}}{c_{2}}$, we conclude that the G-stochastic system $(0.2)$ is pth moment exponentially stable with respect to $x_{1}$.

In what follows, we provide the conditions under which the pth moment exponential stability with respect to a part of the variables of the trivial solution to such a G-SDEs implies the quasi sure exponential stability with respect to a part of the variables.

Theorem 0.9. Consider the G-stochastic system (0.2), assume that there exists a positive constant $\eta$ such that

$$
\widehat{\mathbb{E}}\left(\left|f_{1}\left(t, x_{1}, x_{2}\right)\right|^{p}+\left|h_{1}\left(t, x_{1}, x_{2}\right)\right|^{p}+\left|g_{1}\left(t, x_{1}, x_{2}\right)\right|^{p}\right)<\eta \widehat{\mathbb{E}}\left(\left|x_{1}\right|^{p}\right), \forall x_{1} \in \mathbb{R}^{b}, \forall x_{2} \in \mathbb{R}^{n-b}, \forall t \geq t_{0} \geq 0 .
$$

Furthermore, we suppose that $x_{2}\left(t, t_{0}, x_{0}\right)$ is quasi surely globally uniformly bounded.

Then, the pth moment exponetial stability with respect to $x_{1}$ of the trivial solution of the G-stochastic system (0.2), implies the quasi sure exponential stability.

Proof. By the definition of the pth moment exponential stability with respect to $x_{1}$, there is a pair of positive constants $\lambda_{1}$ and $\lambda_{2}$ such that

$$
\widehat{\mathbb{E}}\left(\left|x_{1}\left(t ; t_{0}, x_{0}\right)\right|^{p}\right) \leq \lambda_{1}\left|x_{0}\right|^{p} e^{-\lambda_{2}\left(t-t_{0}\right)}, \quad \forall t \geq t_{0} \geq 0
$$

Furthermore, we have

$$
x_{1}(t+s)=x_{1}(t)+\int_{t}^{t+s} f_{1}\left(u, x_{1}(u), x_{2}(u)\right) d u+\int_{t}^{t+s} h_{1}\left(u, x_{1}(u), x_{2}(u)\right) d\langle B\rangle_{u}+\int_{t}^{t+s} g_{1}\left(u, x_{1}(u), x_{2}(u)\right) d B_{u}
$$

Which implies, 
By the sub-additivity of G-expectation, we obtain

$$
\begin{aligned}
& \widehat{\mathbb{E}}\left(\sup _{0 \leq s \leq \tau}\left|x_{1}(t+s)\right|^{p}\right) \\
& \leq 4^{p-1}\left[\widehat{\mathbb{E}}\left|x_{1}(t)\right|^{p}+\widehat{\mathbb{E}}\left(\int_{t}^{t+s}\left|f_{1}\left(u, x_{1}(u), x_{2}(u)\right)\right| d u\right)^{p}+\widehat{\mathbb{E}}\left(\sup _{0 \leq s \leq \tau}\left|\int_{t}^{t+s} g_{1}\left(u, x_{1}(u), x_{2}(u)\right) d B_{u}\right|^{p}\right)\right. \\
& \left.+\widehat{\mathbb{E}}\left(\sup _{0 \leq s \leq \tau}\left|\int_{t}^{t+s} h_{1}\left(u, x_{1}(u), x_{2}(u)\right) d\langle B\rangle_{u}\right|^{p}\right)\right] .
\end{aligned}
$$

On account of (0.13), (0.14), and by Hölder's inequality (2010), we obtain

$$
\begin{aligned}
\widehat{\mathbb{E}}\left(\int_{t}^{t+\tau}\left|f_{1}\left(u, x_{1}(u), x_{2}(u)\right)\right| d u\right)^{p} & \leq \tau^{p} \int_{t}^{t+\tau} \widehat{\mathbb{E}}\left|f_{1}\left(u, x_{1}(u), x_{2}(u)\right)\right|^{p} d u \\
& \leq \frac{\lambda_{1}}{\lambda_{2}} \eta \tau^{p}\left|x_{0}\right|^{p} e^{-\lambda_{2}\left(t-t_{0}\right)} .
\end{aligned}
$$

On the other hand, by (0.13), (0.14), and Lemma (0.4), we obtain

$$
\begin{aligned}
\widehat{\mathbb{E}}\left(\sup _{0 \leq s \leq \tau}\left|\int_{t}^{t+\tau} g_{1}\left(u, x_{1}(u), x_{2}(u)\right) d B_{u}\right|^{p}\right) & \leq C_{p} \tau^{\frac{p}{2}-1} \int_{t}^{t+\tau} \widehat{\mathbb{E}}\left|g_{1}\left(u, x_{1}(u), x_{2}(u)\right)\right|^{p} d u \\
& \leq \frac{\lambda_{1}}{\lambda_{2}} C_{p} \eta \tau^{\frac{p}{2}}\left|x_{0}\right|^{p} e^{-\lambda_{2}\left(t-t_{0}\right)} .
\end{aligned}
$$


Likewise, by Lemma (0.3) we obtain

$$
\begin{aligned}
\widehat{\mathbb{E}}\left(\sup _{0 \leq s \leq \tau}\left|\int_{t}^{t+\tau} h_{1}\left(u, x_{1}(u), x_{2}(u)\right) d\langle B\rangle_{u}\right|^{p}\right) & \leq C_{p}^{\prime} \tau^{p-1} \int_{t}^{t+\tau} \widehat{\mathbb{E}}\left|h_{1}\left(u, x_{1}(u), x_{2}(u)\right)\right|^{p} d u \\
& \leq \frac{\lambda_{1}}{\lambda_{2}} C_{p}^{\prime} \eta \tau^{p}\left|x_{0}\right|^{p} e^{-\lambda_{2}\left(t-t_{0}\right)},
\end{aligned}
$$

where, $C_{p}^{\prime}$ is a positive constant dependent only on $\mathrm{p}$.

We conclude from the above inequalities (0.16), (0.17), and (0.18) that

$$
\widehat{\mathbb{E}}\left(\sup _{0 \leq s \leq \tau}\left|x_{1}(t+s)\right|^{p}\right) \leq R e^{-\lambda_{2} t},
$$

where, $R=4^{p-1} \frac{\lambda_{1}}{\lambda_{2}}\left|x_{0}\right|^{p}\left(\lambda_{2}+\eta \tau^{p}\left(1+C_{p} \tau^{-\frac{p}{2}}+C_{p}^{\prime}\right)\right)$.

Now, let $\varepsilon \in\left(0, \lambda_{2}\right)$ be arbitrary. Thanks to Lemma (0.2), we have

By the Borel-Cantelli Lemma for the capacity (0.1), we see that there exists $n_{0}:=n_{0}(\omega)$, such that for almost all $\omega \in \Omega, n>n_{0}(\omega)$,

$$
\sup _{0 \leq s \leq \tau}\left|x_{1}(t+s)\right|^{p} \leq e^{-\left(\lambda_{2}-\varepsilon\right) n \tau}, \text { q.s. }
$$

Where, $t \in[n \tau,(n+1) \tau]$. Then, we obtain

$$
\frac{1}{t} \log \left(\left|x_{1}(t)\right|\right)=\frac{1}{p t} \log \left(\left|x_{1}(t)\right|^{p}\right) \leq-\frac{\left(\lambda_{2}-\varepsilon\right) n \tau}{p n \tau}, \text { q.s. }
$$

Hence, $\lim _{t \rightarrow \infty} \sup \frac{1}{t} \log \left(\left|x_{1}(t)\right|\right) \leq-\frac{\left(\lambda_{2}-\varepsilon\right)}{p}$, q.s.

Since $\varepsilon>0$ is arbitrary, we obtain the desired result

$$
\lim _{t \rightarrow \infty} \sup \frac{1}{t} \log \left(\left|x_{1}(t)\right|\right) \leq-\frac{\lambda_{2}}{p}, \quad \text { q.s. }
$$

That is, the trivial solution of the G-stochastic (0.2) system is quasi surely exponentially stable with respect to $x_{1}$.

Which completes the proof. 


\section{Exponential stability of G-stochastic perturbed differential equations with respect to a part of the variables}

In this section, we consider the following linear stochastic system:

$$
d x(t)=A x(t) d t, \quad \forall t \geq t_{0} \geq 0,
$$

where,

$$
A=(
$$

$\begin{array}{cc}A_{1} & 0 \\ 0 & A_{2}\end{array}, \quad x:=\left(x_{1}, x_{2}\right) \in \mathbb{R}^{b} \times \mathbb{R}^{n-b}, 1 \leq b \leq n$.

- $A_{1}$ is a constant $b \times b$ matrix.

- $A_{2}$ is a constant $(n-b) \times(n-b)$ matrix.

The above system (0.19) might be regarded as the following system:

$d x_{1}(t)=A_{1} x_{1}(t) d t$

$d x_{2}(t)=A_{2} x_{2}(t) d t$, $(0.20)$

with initial condition $x\left(t_{0}\right):=x_{0}:=\left(x_{1_{0}}, x_{2_{0}}\right) \in \mathbb{R}^{b} \times \mathbb{R}^{n-b}$.

Assume that some parameters are excited or perturbed by G-Brownian motion, and the perturbed system has the form:

$d x_{1}(t)=A_{1} x_{1}(t) d t+g\left(t, x_{1}(t), x_{2}(t)\right) d B_{t}$

$d x_{2}(t)=A_{2} x_{2}(t) d t$

$(0.21)$

with the same initial conditions, where $B_{t}=\left(B_{1}(t), \ldots, B_{m}(t)\right)^{T}$ is an $m$-dimensional G-Brownian motion, and $g: \mathbb{R}^{+} \times \mathbb{R}^{b} \times \mathbb{R}^{n-b} \longrightarrow \mathbb{R}^{b \times m}$. 
Assume that conditions of existence and uniqueness of solutions are satisfied, see $(2006)$. Denote by $x\left(t, t_{0}, x_{0}\right)=$ $\left(x_{1}\left(t ; t_{0}, x_{0}\right), x_{2}\left(t ; t_{0}, x_{0}\right)\right)$ the solution of system $(0.21)$.

We suppose that the origin of the linear stochastic system $(0.20)$ is quasi surely exponentially stable with respect to $x_{1}$. Furthermore, we assume that the origin $x=(0,0)$ is an equilibrium point of the G-stochastic perturbed system $(0.21)$, that is $g(t, 0,0)=0$ for all $t \geq 0$.

The objective of this section is to give sufficient conditions under which the G-stochastic perturbed system (0.21) is still quasi surely exponentially stable with respect to $x_{1}$.

Theorem 0.10. Let $\lambda_{1}$ be the maximum of the real parts of all eigenvalues of $-A_{1}$.

Suppose there exist a constant $c_{1} \geq 0$, and a polynomial $p_{1}(t)$ such that for all $x_{1} \in \mathbb{R}^{b}, x_{2} \in \mathbb{R}^{n-b}$, and sufficiently large $t$,

$$
\left|g\left(t, x_{1}(t), x_{2}(t)\right)\right|^{2} \leq p_{1}(t) e^{\left(-2 \lambda_{1}+c_{1}\right) t}, \quad q . s .
$$

Furthermore, we assume that $\lim _{t \rightarrow \infty} \sup \frac{\log \left|e^{A_{1} t}\right|^{2}}{t} \leq-c_{2}$, where, $c_{2}$ is a positive constant.

Moreover, we suppose that $x_{2}\left(t, t_{0}, x_{0}\right)$ is quasi surely globally uniformly bounded.

Then,

$\lim _{t \rightarrow \infty} \sup \frac{\log \left|x_{1}\left(t ; t_{0}, x_{0}\right)\right|^{2}}{t} \leq-\left(c_{2}-c_{1}\right)$, q.s. for all $t_{0} \geq 0$ and $x_{0} \in \mathbb{R}^{n}$.

In particular, if $c_{2}>c_{1}$, then the G-stochastic perturbed system (0.21) is said to be quasi surely exponentially stable with respect to $x_{1}$.

In order to prove this theorem, let us start by recalling an important Gronwall Lemma (S. S. Dragomir \& applications, 2003), which will be very useful later on.

Lemma 0.11. Let $b(t), c(t)$, and $u(t)$ be continuous functions for $t \geq t_{0} \geq 0$, let $b(t)$ be nonnegative for $t \geq t_{0} \geq 0$, $\phi$ is a constant and suppose

$$
u(t) \leq \phi+\int_{t_{0}}^{t}[b(s) u(s)+c(s)] d s, \quad t \geq t_{0} \geq 0 .
$$

Then,

$$
u(t) \leq \phi \exp \left(\int_{t_{0}}^{t} b(\tau) d \tau\right)+\int_{t_{0}}^{t} c(s) \exp \left(\int_{s}^{t} b(\tau) d \tau\right) d s, \quad t \geq t_{0} \geq 0
$$

We also need the following Lemma (1002), which has its own importance.

Lemma 0.12. Let $B_{t}$ be a one-dimensional G-Brownian motion. Suppose that there exist constants $\varepsilon>0$ and $\alpha>0$ such that

$$
\widehat{\mathbb{E}}\left(\exp \left[\frac{\alpha^{2}}{2}(1+\varepsilon) \int_{0}^{T} g^{2}(s) d\langle B\rangle_{s}\right]\right)<\infty, \quad \forall g \in M_{G}^{2}(0, T) .
$$

Then, for any $T>0$ and $\beta>0$, 


$$
\hat{C}\left(\sup _{0 \leq t \leq T}\left[\int_{0}^{t} g(s) d B_{s}-\frac{\alpha}{2} \int_{0}^{t} g^{2}(s) d\langle B\rangle_{s}\right]>\beta\right) \leq \exp (-\alpha \beta) .
$$

Now, we are able to prove our theorem.

Proof of Theorem 0.10. Fix $\epsilon>0$ arbitrarly, and there exists $\rho=\rho(\epsilon)$ such that

$$
\left|e^{-A_{1} t}\right|^{2} \leq \rho e^{\left(2 \lambda_{1}+\epsilon\right) t}, \quad p_{1}(t) \leq \rho e^{\epsilon t}, \quad t>0 .
$$

By G-Itô's formula, we obtain

$$
d\left(e^{-A_{1} t} x_{1}(t)\right)=e^{-A_{1} t} g\left(t, x_{1}(t), x_{2}(t)\right) d B_{t} .
$$

Define $W(t)=\left|e^{-A_{1} t} x_{1}(t)\right|^{2}$. By G-Itô's formula again,

$$
\begin{gathered}
W(t)=W\left(t_{0}\right)+2 \int_{t_{0}}^{t} x_{1}^{T}(s) e^{-A_{1}^{T} s} e^{-A_{1} s} g\left(s, x_{1}(s), x_{2}(s)\right) d B_{s} \\
+\int_{t_{0}}^{t} \operatorname{trace}\left(e^{-A_{1} s} g\left(s, x_{1}(s), x_{2}(s)\right) g_{1}^{T}\left(s, x_{1}(s), x_{2}(s)\right) e^{-A_{1}^{T} s}\right) d\langle B\rangle_{s} .
\end{gathered}
$$

It follows from Lemma (0.12) that for any $\alpha>0, \beta>0$, and $\tau>t_{0}$.

$$
\begin{aligned}
\hat{C}\left(\operatorname { s u p } _ { t _ { 0 } \leq t \leq \tau } \left[\int_{t_{0}}^{t} x_{1}^{T}(s) e^{-A_{1}^{T} s} e^{-A_{1} s} g\left(s, x_{1}(s), x_{2}(s)\right) d B_{s}-\frac{\alpha}{2}\right.\right. \\
\left.\left.\quad \int_{t_{0}}^{t} x_{1}^{T}(s) e^{-A_{1}^{T} s} e^{-A_{1} s} g\left(s, x_{1}(s), x_{2}(s)\right) g_{1}^{T}\left(s, x_{1}(s), x_{2}(s)\right) e^{-A_{1}^{T} s} e^{-A_{1} s} x_{1}(s) d\langle B\rangle_{s}\right]>\beta\right) \leq \exp (-\alpha \beta) .
\end{aligned}
$$

Choose an arbitrary $\theta>1$, and let $k$ be an integer large enough so that $k>t_{0}$. Set

$$
\alpha=e^{-c_{1} k}, \quad \beta=\theta e^{c_{1} k} \log k, \quad \tau=k .
$$

Then, we obtain 
Applying the Borel-Cantelli Lemma for the capacity, we see that for almost all $\omega \in \Omega$, there exists $k_{0}=k_{0}(\omega)$ such that

for all $k>k_{0}, \quad t_{0} \leq t \leq k$. By using condition (0.22), it follows that

This together with (0.23), we obtain

In chapter III of Peng (1002), we have for each $0 \leq s \leq t \leq T$,

$$
\langle B\rangle_{t}-\langle B\rangle_{s} \leq \bar{\sigma}^{2}(t-s) .
$$

Based on this fact and the inequality (??), we obtain

$$
W(t) \leq W\left(t_{0}\right)+e^{-c_{1} k} \rho^{2} \bar{\sigma}^{2} \int_{t_{0}}^{t} W(s) e^{c_{1} s} d s+2 \theta e^{c_{1} k} \log k+b \rho^{2} \bar{\sigma}^{2} \int_{t_{0}}^{t} e^{c_{1} s} d s .
$$

Thanks to Lemma (0.11), we have

That is,

$$
W(t) \leq\left(W\left(t_{0}\right)+2 \theta e^{c_{1} k} \log k+\frac{b \rho^{2} \bar{\sigma}^{2}}{c_{1}} e^{c_{1} k}\right) \exp \left(\frac{\rho^{2} \bar{\sigma}^{2}}{c_{1}}\right), \quad t_{0} \leq t \leq k, \quad k \geq k_{0}, \quad \text { q.s. }
$$


Since $\theta>1$ is arbitrary and $\frac{W(t)}{e^{c_{1} t} \log t} \leq \frac{W(t)}{e^{c_{1}(k-1)} \log (k-1)}, \quad k-1 \leq t \leq k$.

From (0.24), we see immediately that

Since,

$$
\lim _{t \longrightarrow \infty} \sup \frac{\log \left|x_{1}\left(t ; t_{0}, x_{0}\right)\right|^{2}}{t} \leq \lim _{t \longrightarrow \infty} \sup \frac{\log \left|e^{A t}\right|^{2}}{t}+\lim _{t \longrightarrow \infty} \sup \frac{\log \left|e^{-A t} x_{1}\left(t ; t_{0}, x_{0}\right)\right|^{2}}{t}
$$

Consequently,

$\lim _{t \rightarrow \infty} \sup \frac{\log \left|x_{1}\left(t ; t_{0}, x_{0}\right)\right|^{2}}{t} \leq-c_{2}+c_{1}=-\left(c_{2}-c_{1}\right)$, q.s.

If the inequality $c_{2}>c_{1}$ is satisfied, then the G-stochastic perturbed system (0.21) is quasi surely exponentially stable with respect to $x_{1}$.

\section{Examples}

The following illustrative examples are provided to show the usefulness of the obtained results.

Example 0.13. Consider the following G-stochastic system:

$d x_{1}(t)=-2 x_{1} d t-\frac{1}{2} \sin ^{2}\left(x_{2}\right) e^{-2 t} x_{1} d\langle B\rangle_{t}+\left(1+e^{-t}\left|\sin \left(x_{2}\right)\right|\right) x_{1} d B_{t}$

$d x_{2}(t)=2 \cos (t) x_{2} d t$

$(0.25)$

where, $x=\left(x_{1}, x_{2}\right)^{T} \in \mathbb{R}^{2}, B$ is a one-dimension G-Brownian motion and $B=N\left(0 \times\left[\frac{1}{2}, 1\right]\right)$, with initial value $x_{0}=\left(x_{1_{0}}, x_{2_{0}}\right)$.

This system has the trivial solution $x_{1}=0, x_{2}=0$.

It is clear that $x_{2}(t)$ is quasi surely globally uniformly bounded. In fact, for all $t \geq t_{0} \geq 0$, and all $x_{2_{0}} \in \mathbb{R}$ with $\left|x_{2_{0}}\right| \leq \beta$, we have $\left|x_{2}(t)\right| \leq \beta e^{2 \sin (t)}$ q.s.

Denote $V=x_{1}^{2}$, then we have 
By the sub-additivity of the function $G$, we obtain

That is,

$$
L V(t, x) \leq(-3+\varphi(t)) x_{1}^{2},
$$

where $\varphi(t)=2 e^{-t}$, which satisfies condition (0.9) of Theorem (0.7).

Hence, all conditions of Theorem (0.7) are fulfilled with $p=2$ and then the G-stochastic system (0.25) is pth moment exponentially stable with respect to $x_{1}$. Furthermore, we have

1. $\left|f_{1}(t, x)\right|^{2}=4\left|x_{1}\right|^{2}$,

2. $\left|h_{1}(t, x)\right|^{2}=\left|\frac{1}{2} \sin ^{2}\left(x_{2}\right) e^{-2 t} x_{1}\right|^{2} \leq\left|x_{1}\right|^{2}$,

3. $\left|g_{1}(t, x)\right|^{2}=\left|\left(1+e^{-t}\left|\sin \left(x_{2}\right)\right|\right) x_{1}\right|^{2} \leq 2\left(1+e^{-2 t}\left|\sin \left(x_{2}\right)\right|^{2}\right)\left|x_{1}\right|^{2} \leq 4\left|x_{1}\right|^{2}$.

Then, we obtain

$$
\hat{\mathbb{E}}\left(\left|f_{1}(t, x)\right|^{2}+\left|h_{1}(t, x)\right|^{2}+\left|g_{1}(t, x)\right|^{2}\right) \leq 9 \hat{\mathbb{E}}\left(\left|x_{1}\right|^{2}\right) .
$$

Hence, from Theorem (0.9), with $p=2$ and $\eta=9$, one can deduce that the G-stochastic system (0.25) is quasi sure exponential stable with respect to $x_{1}$.

Example 0.14. Consider the following G-stochastic system

$d x_{1}(t)=-2 x_{1}(t) d t+\left(1+t^{2}\right) e^{-4.5 t} \frac{x(t)}{1+|x(t)|} d B_{t}$

$d x_{2}(t)=A_{2} x_{2}(t) d t$ 
where, $x=\left(x_{1}, x_{2}\right) \in \mathbb{R}^{3}, x_{2}=\left(z_{1}, z_{2}\right) \in \mathbb{R}^{2}$ and $B(t)$ is one-dimensional G-Brownian motion,

$$
A_{2}=(
$$

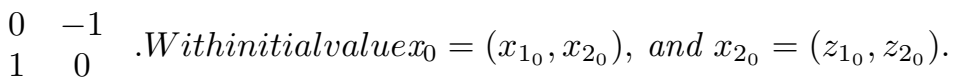

By simple resolution, we obtain

$$
x_{2}(t)=(
$$

$z_{1_{0}}(t, w) \cos (t)-z_{2_{0}}(t, w) \sin (t)$
$z_{1_{0}}(t, w) \sin (t)+z_{2_{0}}(t, w) \cos (t)$ .Usingthe2-normleadsto $\left|x_{2}(t)\right|=\left|x_{2_{0}}\right|$. Itisclearthat $x_{2}(t)$ is quasi surely globally uniformly bounded. In fact, for all $t \geq t_{0} \geq 0$, and all $x_{2_{0}} \in \mathbb{R}^{2}$ with $\left|x_{2_{0}}\right| \leq \alpha^{\prime}$, we have $\left|x_{2}(t)\right| \leq \alpha^{\prime} \quad$ q.s It is clear that, the constants of Theorem (0.10) are $c_{1}=0.5$, and $c_{2}=2$.

Hence, by Theorem (0.10) we deduce that $\limsup _{t \rightarrow \infty} \frac{\log \left|x_{1}\left(t, t_{0}, x_{0}\right)\right|^{2}}{t} \leq-1.5$ q.s. for all $t_{0} \geq 0$ and $x_{0} \in \mathbb{R}^{2}$. Indeed, the G-stochastic perturbed system (0.26) is quasi sure exponential stable with respect to $x_{1}$.

\section{References}

(1982).

(1980).

(1969).

(1957).

(1989).

(2006).

(1002).

(2013).

(2014).

(2008).

(2010).

(2013).

(2016).

(2003). 